Теорія Ймовір. та Матем. Статист. Вип. 76, 2007
Theor. Probability and Math. Statist.

No. 76, 2008, Pages 159-167

S 0094-9000(08)00740-0

Article electronically published on July 17, 2008

\title{
THE DISTRIBUTION OF A RANDOM SUM OF EXPONENTIALS WITH AN APPLICATION TO A TRAFFIC PROBLEM
}

UDC 519.21

FRANK RECKER

\begin{abstract}
We study a random sum of exponentially distributed random variables. The stopping time is defined to be the first realization that is greater than or equal to a given constant. We will derive an expression for the distribution function of this sum. This has applications in determining the waiting time for a large gap in a Poisson process. As an example, we will give a traffic problem, where such a waiting time occurs.
\end{abstract}

\section{INTRODUCTION}

In traffic situations one sometimes has to wait for a gap to occur. Think of a person crossing a street or a car on a minor road that wants to enter a main road. We assume that the cars on the main road arrive with exponentially distributed inter-arrival times and one has to wait until the distance between two consecutive cars is large enough, i.e. greater than or equal to $\tau$. The distribution of the waiting time depends on the traffic intensity $\lambda>0$ and the minimal gap $\tau>0$. Mathematically, we have a sequence of r.v.s $\left(X_{n}\right)_{n \in \mathbb{N}}$, such that the $X_{n}$ are i.i.d. and each $X_{n}$ is $\operatorname{Exp}(\lambda)$-distributed (exponentially with parameter $\lambda$ ). The stopping time $T$ is defined as $T:=\min \left\{n \in \mathbb{N} \mid X_{n} \geq \tau\right\}$. The waiting time is the r.v.

$$
Z:=\sum_{n=1}^{T-1} X_{n}
$$

with the usual convention $\sum_{n=1}^{0} \cdots=0$.

In the following, the distribution of $Z$ will be derived. We will show that the distribution function $F_{Z}$ of $Z$ can be described by polynomials. Furthermore, we will derive a recursive formula for the coefficients of the polynomials.

The above-defined waiting time occurs in the context of queuing-problems from real world traffic, inspired by $[2,3$. On a minor road, the cars arrive at the end of the queue with traffic intensity $\mu$ and cars leave the queue when the gap in the traffic on the major road is large enough. The qualitative behavior of this queue is analyzed in [5. There it is shown that there exists a sharp bound $\mu^{*}$ such that the queue-length is recurrent for $\mu<\mu^{*}$ and that it is transient for $\mu>\mu^{*}$. In [5] we used the technique of the embedded Markov chain and we did not need the distribution of the service time $Z$. Nevertheless, knowledge of this distribution is useful, if one wants to apply standard techniques from queuing theory, as for instance deriving quantitative statements about the ergodic distribution of the queue length (cf. [1, 4]).

2000 Mathematics Subject Classification. Primary 60G40, 90B20.

Key words and phrases. Poisson process, stopping time, queuing theory, traffic problems. 
We will give the exact definitions of the model and the theorems in Section 2 The main parts of the proof will be given in Section 3. The technical calculations are postponed to Section 4 .

\section{The Distribution of the WAiting time}

We assume that all random variables are defined on a probability space $(\Omega, \mathcal{A}, \mathrm{P})$. We use $\mathbb{N}$ for the set $\{1,2, \ldots\}$ and $\mathbb{N}_{0}$ for $\{0,1, \ldots\}$. The next definition summarizes the model given in the introduction:

Definition 2.1. Let $\lambda, \tau \in \mathbb{R}, \lambda, \tau>0$, and let $\left(X_{n}\right)_{n \in \mathbb{N}}$ be a sequence of i.i.d. $\operatorname{Exp}(\lambda)$ distributed random variables. Further let $T$ be the stopping time

$$
T:=\min \left\{n \in \mathbb{N} \mid X_{n} \geq \tau\right\} .
$$

Then the waiting time for intensity $\lambda$ and minimal gap $\tau$ is the random variable

$$
Z:=\sum_{n=1}^{T-1} X_{n} .
$$

Since $T$ is a stopping time, $Z$ is indeed measurable. Before we proceed we give a remark on the term intensity: In renewal theory, the intensity is defined as the reciprocal of the expected inter-arrival time. In our case, the renewal process is a Poisson process and hence this value is exactly that of the parameter $\lambda$.

We write $F_{Z}$ for the distribution function of $Z$, i.e. $F_{Z}(x)=\mathrm{P}(\{Z \leq x\})$ for all $x \in \mathbb{R}$. We will now state the theorems concerning the distribution function of the waiting time.

Theorem 2.2. Let $Z$ be the waiting time for the parameters $\lambda, \tau>0$. Then $F_{Z}(x)=0$ for all $x<0$. Further for each $n \in \mathbb{N}$ the restriction of $F_{Z}$ on the interval $[(n-1) \tau, n \tau]$ is a polynomial of degree $n$. Finally $F_{Z}$ has only one jump point at 0 (and hence $F_{Z}$ is continuous at all other points of $\mathbb{R}$ ).

The next theorem states how the polynomials for $F_{Z}$ can be calculated.

Theorem 2.3. Let $\lambda, \tau, Z$, and $F_{Z}$ be as in Theorem 2.2. For each $n \in \mathbb{N}$ define the polynomial $p_{n}$ as

$$
p_{n}(x)=\sum_{k=0}^{n} a_{n, k} x^{k},
$$

where the coefficients are defined as follows:

$$
\begin{gathered}
a_{1,0}=e^{-\lambda \tau}, \\
a_{1,1}=\lambda e^{-\lambda \tau},
\end{gathered}
$$

and for each $n \geq 2$ :

$$
\begin{gathered}
a_{n, 0}=\sum_{k=0}^{n-2}\left((-1)^{k} \frac{1}{k !}(\lambda \tau)^{k} e^{-k \lambda \tau} a_{n-1-k, 0}\right)-\sum_{k=1}^{n-1}\left((-1)^{k} \frac{1}{k !}(\lambda \tau)^{k} e^{-k \lambda \tau}\right), \\
a_{n, k}=(-1)^{k+1} \frac{1}{k !} \lambda^{k} e^{-k \lambda \tau}\left(1-a_{n-k, 0}\right)
\end{gathered}
$$

for each $k=1, \ldots, n-1$, and

$$
a_{n, n}=(-1)^{n+1} \frac{1}{n !} \lambda^{n} e^{-n \lambda \tau} .
$$

Then for each $n \in \mathbb{N}$ and each $x \in[(n-1) \tau, n \tau]$ :

$$
F_{Z}(x)=p_{n}(x-(n-1) \tau) .
$$


Note that for each $n \geq 2$ and $k=0, \ldots, n$, the definition of $a_{n, k}$ uses only $a_{n^{\prime}, k^{\prime}}$ with $n^{\prime}<n$. Hence the definition is indeed recursive.

Theorem 2.3 gives the value of $F_{Z}(x)$ for all $x \in \mathbb{R}$. We have $F_{Z}(x)=0$ for all $x<0$. For $x \geq 0$ chose $n \in \mathbb{N}$ with $(n-1) \tau \leq x \leq n \tau$. Then $F_{Z}(x)=p_{n}(x-(n-1) \tau)$. Observe that if $x$ is a multiple of $\tau$, then the theorem claims that both possible values for $n$ deliver the same result.

Of course $q_{n}(x):=p_{n}(x-(n-1) \tau)$ is also a polynomial of degree $n$ and we get $F_{Z}(x)=q_{n}(x)$ for the above chosen $n$. But since the coefficients of $q_{n}$ have a much more complicated recursive formula, we decided to use the $p_{n}$ defined above. Figure 1 shows a part of the graph of $F_{Z}$ for the parameters $\lambda=0.5$ and $\tau=3.0$.

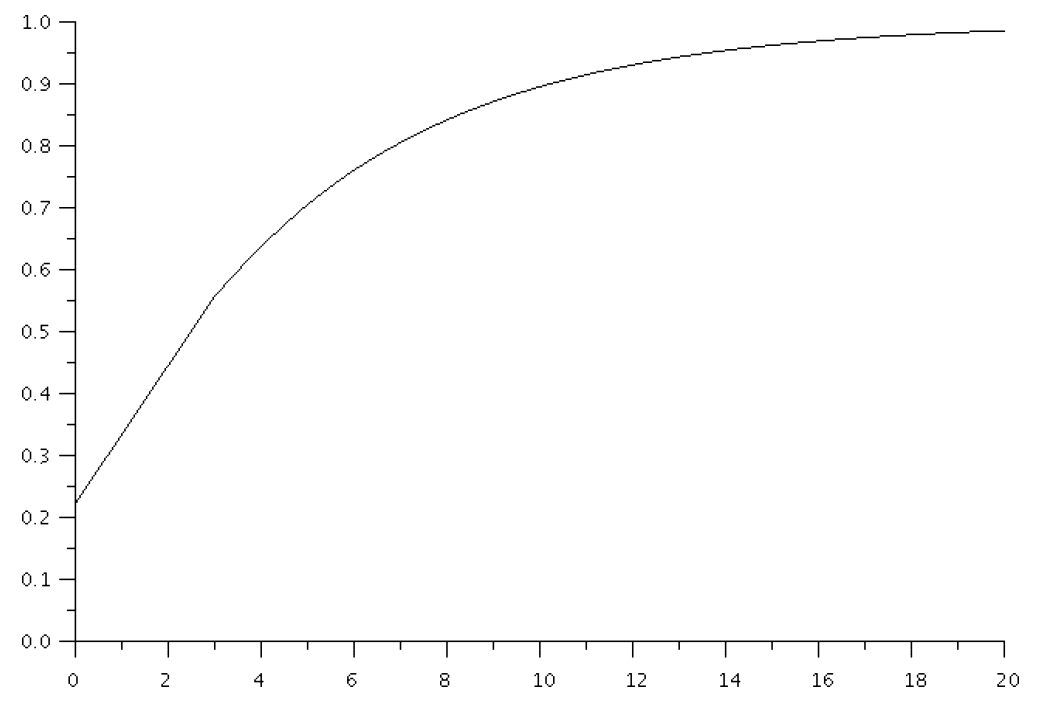

Figure 1. The distribution function $F_{Z}$ for $\lambda=0.5$ and $\tau=3.0$

\section{THE PROOF}

The proof consists of three steps. First, we will show directly in Lemma 3.1 that the theorems are true for all $x \in(-\infty, \tau]$. After that we prove in Lemmas 3.2 and 3.3 that the distribution function can be characterized by an integral equation. Finally, we show in Lemma 3.4 that the polynomials of Theorem 2.3 fulfill this integral equation.

In this whole chapter let $\lambda, \tau,\left(X_{n}\right)_{n \in \mathbb{N}}, T, Z, F_{Z},\left(p_{n}\right)_{n \in \mathbb{N}}$, and $\left(a_{n, k}\right)_{n \in \mathbb{N}, k \in\{0, \ldots, n\}}$ be defined as in Definition 2.1 and Theorems 2.2 and 2.3

Lemma 3.1. For all $x<0, F_{Z}(x)=0$. For all $x \in[0, \tau]$ we have

$$
F_{Z}(x)=(1+\lambda x) e^{-\lambda \tau} \text {. }
$$

Proof. Case $x<0$ : The r.v. $Z$ is by (2.1) non-negative almost surely. Hence

$$
F_{Z}(x)=\mathrm{P}(\{Z \leq x\})=0 .
$$

Case $x=0$ : For each $n \in \mathbb{N}$ we have $\mathrm{P}\left(X_{n}=0\right)=0$. Hence we get

$$
F_{Z}(0)=\mathrm{P}(\{Z=0\})=\mathrm{P}(\{T=1\})=\mathrm{P}\left(\left\{X_{1} \geq \tau\right\}\right)=e^{-\lambda \tau} .
$$

Case $x \in(0, \tau)$ : We have

$$
\mathrm{P}(\{Z \leq x\})=\sum_{n=1}^{\infty} \mathrm{P}\left(\left\{X_{1}+\cdots+X_{n-1} \leq x, T=n\right\}\right) .
$$


The term for $n=1$ is $e^{-\lambda \tau}$, as above. For $n \geq 2$ we have

$$
\mathrm{P}\left(\left\{X_{1}+\cdots+X_{n-1} \leq x, T=n\right\}\right)=\mathrm{P}\left(\left\{X_{1}+\cdots+X_{n-1} \leq x, X_{n} \geq \tau\right\}\right),
$$

since $x<\tau$ (which is crucial and makes it hard to determine the distribution function for $x>\tau)$. Now $X_{n}$ is independent from $X_{1}, \ldots, X_{n-1}$. Thus

$$
\mathrm{P}\left(\left\{X_{1}+\cdots+X_{n-1} \leq x, X_{n} \geq \tau\right\}\right)=e^{-\lambda \tau} \mathrm{P}\left(\left\{X_{1}+\cdots+X_{n-1} \leq x\right\}\right) .
$$

The sum $X_{1}+\cdots+X_{n-1}$ is distributed according to the Erlang distribution; i.e., it has the Lebesgue density

$$
y \mapsto \lambda^{n-1} \frac{y^{n-2}}{(n-2) !} e^{-\lambda y}
$$

for all $y \geq 0$. Therefore

$$
\mathrm{P}\left(\left\{X_{1}+\cdots+X_{n-1} \leq x\right\}\right)=\int_{0}^{x} \lambda^{n-1} \frac{y^{n-2}}{(n-2) !} e^{-\lambda y} d y .
$$

It follows by the Monotone Convergence Theorem that

$$
\begin{aligned}
\mathrm{P}(\{Z \leq x\}) & =e^{-\lambda \tau}+e^{-\lambda \tau} \sum_{n=2}^{\infty}\left(\int_{0}^{x} \lambda^{n-1} \frac{y^{n-2}}{(n-2) !} e^{-\lambda y} d y\right) \\
& =e^{-\lambda \tau}+e^{-\lambda \tau} \int_{0}^{x}\left(\sum_{n=2}^{\infty} \lambda^{n-1} \frac{y^{n-2}}{(n-2) !} e^{-\lambda y}\right) d y \\
& =e^{-\lambda \tau}+e^{-\lambda \tau} \int_{0}^{x} \lambda d y \\
& =e^{-\lambda \tau}+\lambda x e^{-\lambda \tau}
\end{aligned}
$$

Case $x=\tau$ : We have $\mathrm{P}(\{Z=\tau\})=0$, since $\tau>0$ and all $X_{i}$ are Lebesgue-continuous. Thus $F_{Z}$ is continuous in $\tau$ and we have by the already proven cases,

$$
F_{Z}(\tau)=\lim _{x \nearrow \tau} F_{Z}(x)=e^{-\lambda \tau}+\lambda \tau e^{-\lambda \tau} .
$$

Now we come to the second step of the proof. The next lemma shows that the distribution function $F_{Z}$ fulfills an integral equation.

Lemma 3.2. For all $x \geq \tau$ we have

$$
F_{Z}(x)=e^{-\lambda \tau}+\int_{0}^{\tau} F_{Z}(x-y) \lambda e^{-\lambda y} d y .
$$

Proof. Define $T^{\prime}:=\min \left\{n \geq 2 \mid X_{n} \geq \tau\right\}$ and $Z^{\prime}:=\sum_{n=2}^{T^{\prime}-1} X_{n}$. The r.v.s $\left(X_{n}\right)_{n \in \mathbb{N}}$ are i.i.d. Hence $Z^{\prime}$ has the same distribution as $Z$. Furthermore, for all $\omega \in \Omega$ we have: If $X_{1}(\omega)<\tau$, then $T^{\prime}(\omega)=T(\omega)$ and $Z(\omega)=X_{1}(\omega)+Z^{\prime}(\omega)$. Using this, we get

$$
\mathrm{P}(\{Z \leq x\})=\mathrm{P}\left(\left\{X_{1} \geq \tau\right\}\right)+\mathrm{P}\left(\left\{X_{1}<\tau ; X_{1}+Z^{\prime} \leq x\right\}\right) .
$$

As above, we have $\mathrm{P}\left(X_{1} \geq \tau\right)=e^{-\lambda \tau}$. For the second term, we use $\mathrm{P}\left(\left\{X_{1}=\tau\right\}\right)=0$ and thus

$$
\begin{aligned}
\mathrm{P}\left(\left\{X_{1}<\tau ; X_{1}+Z^{\prime} \leq x\right\}\right) & =\int_{0}^{\tau} \mathrm{P}\left(\left\{Z^{\prime} \leq x-y\right\}\right) P_{X_{1}}(d y) \\
& =\int_{0}^{\tau} F_{Z}(x-y) \lambda e^{-\lambda y} d y .
\end{aligned}
$$

In fact, $F_{Z}$ is already characterized by (3.1) and the values for all $x \leq \tau$, as follows from Lemma 3.3 
Lemma 3.3. Let $f, g: \mathbb{R} \rightarrow \mathbb{R}$ be integrable functions with $f(x)=g(x)$ for all $x \leq \tau$ and

$$
f(x)=e^{-\lambda \tau}+\int_{0}^{\tau} f(x-y) \lambda e^{-\lambda y} d y,
$$

respectively

$$
g(x)=e^{-\lambda \tau}+\int_{0}^{\tau} g(x-y) \lambda e^{-\lambda y} d y
$$

for all $x>\tau$. If $f$ and $g$ are further bounded an all compact sets, then $f=g$.

Proof. Define $h=f-g$. For all $x>\tau$, we have

$$
h(x)=\int_{0}^{\tau} h(x-y) \lambda e^{-\lambda y} d y .
$$

Since $h(x)=0$ for all $x \leq \tau$, in fact (3.3) holds for all $x \in \mathbb{R}$.

We first prove that $h$ is continuous: Let $x, \varepsilon \in \mathbb{R}$ be arbitrary. Then

$$
\begin{aligned}
h(x+\varepsilon) & =\int_{0}^{\tau} h(x+\varepsilon-y) \lambda e^{-\lambda y} d y=\int_{-\varepsilon}^{\tau-\varepsilon} h(x-y) \lambda e^{-\lambda(y+\varepsilon)} d y \\
& =e^{-\lambda \varepsilon}\left(\int_{0}^{\tau} h(x-y) \lambda e^{-\lambda y} d y+\int_{-\varepsilon}^{0} h(x-y) \lambda e^{-\lambda y} d y-\int_{\tau-\varepsilon}^{\tau} h(x-y) \lambda e^{-\lambda y} d y\right) .
\end{aligned}
$$

Since $h$ is bounded on compact sets, the second and the third integral converge towards 0 as $\varepsilon \rightarrow 0$. Since $e^{-\lambda \varepsilon} \rightarrow 1$ for $\varepsilon \rightarrow 0$, we get with (3.3): $\lim _{\varepsilon \rightarrow 0} h(x+\varepsilon)=h(x)$.

Assume now that there exists $x \in \mathbb{R}$ with $h(x) \neq 0$. Since $h(x)=0$ for all $x \leq \tau$, we can choose $x_{0}$ and $x_{1}$ such that $h(x)=0$ for all $x \leq x_{0}, h\left(x_{1}\right) \neq 0$ and $x_{0}<x_{1}<x_{0}+\tau$. W.l.o.g. we assume further that $h\left(x_{1}\right)>0$ (otherwise proceed with $-h$ ). Since $h$ is continuous, $h$ reaches its maximum on $\left[x_{0}, x_{1}\right]$, say at $x_{2}$. Thus

$$
0<h\left(x_{2}\right)=\int_{0}^{\tau} h\left(x_{2}-y\right) \lambda e^{-\lambda y} d y .
$$

For all $y \geq 0$ we have $h\left(x_{2}-y\right) \leq h\left(x_{2}\right)$ ( $x_{2}$ is a maximal point of $h$ in $\left[x_{1}, x_{2}\right]$ and $h(x)=0$ for all $\left.x \leq x_{1}\right)$. Hence

$$
0<h\left(x_{2}\right) \leq h\left(x_{2}\right) \int_{0}^{\tau} \lambda e^{-\lambda y} d y .
$$

This is a contradiction, since the last integral is less than 1 . Hence $h=0$ and thus $f=g$.

The last step towards the proof of the theorems is to show that the $p_{n}$ from Theorem 2.3 fulfill a (3.2)-like equation.

Lemma 3.4 (Recursion Lemma). Let $\left(p_{n}\right)_{n \in \mathbb{N}}$ be defined as in Theorem 2.3. Then

$$
p_{1}(x)=(1+\lambda x) e^{-\lambda \tau}
$$

and for each $n \geq 2$ and each $0 \leq x \leq \tau$,

$$
p_{n}(x)=e^{-\lambda \tau}+\int_{0}^{x} p_{n}(x-y) \lambda e^{-\lambda y} d y+\int_{x}^{\tau} p_{n-1}(x-y+\tau) \lambda e^{-\lambda y} d y .
$$

Furthermore, we have $p_{n}(0)=p_{n-1}(\tau)$.

The calculations for the proof of Lemma 3.4 are quite long. We will give them in the next section. Here we just mention one remarkable thing: Some $e^{-\lambda x}$-terms occur on the right side of (3.5). From this one would assume that the term cannot be a polynomial in $x$. But in fact, the $e^{-\lambda x}$-terms from both integrals just cancel each other (cf. (4.5) and (4.6) $)$. 
Proof of Theorems 2.2 and 2.3. Define $f: \mathbb{R} \rightarrow \mathbb{R}$ as $f(x)=0$ for all $x<0$. For all $x \geq 0$ define $f(x)=p_{n}(x-(n-1) \tau)$, where $n$ is chosen such that $(n-1) \tau \leq x \leq n \tau$ (if $x$ is a multiple of $\tau$, then by Lemma 3.4 both possible $n$ deliver the same result). From (3.4) and Lemma 3.1] it follows that $f(x)=F_{Z}(x)$ for all $x \leq \tau$.

From Lemma 3.4 it follows that $f$ fulfills (3.2) for all $x \geq \tau$. Lemmas 3.2 and 3.3 hence show that $F_{Z}=f$. Finally $f$ is continuous in every $x \in \mathbb{R}, x \neq 0$, since $f(x)=0$ for all $x<0$ and $f$ is also continuous at the glue-points of the polynomials (cf. Lemma 3.4).

It remains to prove Lemma 3.4 This is done in the next section.

\section{The Proof of the ReCURsion lemma}

Lemma 4.1 is obvious. We state it once, since we will use it heavily during the calculations. We denote the $k$-th derivation of a function $f$ by $f^{(k)}$.

Lemma 4.1. Let $p=a_{0}+\cdots+a_{n} x^{n}$ be a polynomial of degree $n \in \mathbb{N}_{0}$. Then for each $k=0, \ldots, n$ we have

$$
p^{(k)}(x)=\sum_{m=0}^{n-k} \frac{(m+k) !}{m !} a_{m+k} x^{m}
$$

and in particular

$$
p^{(k)}(0)=k ! a_{k} .
$$

From now on, we use again the notation from Theorem 2.3 We will build up a bunch of equations for the coefficients $a_{n, k}$, which will eventually prove Lemma 3.4.

Lemma 4.2. For all $n \in \mathbb{N}, n \geq 2$,

$$
\sum_{k=0}^{n}(-1)^{k+1} \frac{1}{\lambda^{k}} k ! a_{n, k}=\sum_{k=0}^{n-1}(-1)^{k+1} \frac{1}{\lambda^{k}} \sum_{m=0}^{n-1-k} \frac{(m+k) !}{m !} a_{n-1, m+k} \tau^{m} .
$$

Proof. We apply (2.5) and (2.6) on the left side of (4.1). This gives

$$
-\sum_{k=0}^{n-1} e^{-k \lambda \tau} a_{n-k, 0}+\sum_{k=1}^{n} e^{-k \lambda \tau}
$$

In this equation we replace $a_{1,0}$ by $e^{-\lambda \tau}$ (cf. (2.2) ) and every other $a_{n-k, 0}$ according to (2.4). Here is the result for the left side of (4.1):

$$
\begin{aligned}
& -\sum_{k=0}^{n-2} \sum_{m=0}^{n-k-2}(-1)^{m} \frac{1}{m !}(\lambda \tau)^{m} e^{-(m+k) \lambda \tau} a_{n-k-m-1,0} \\
& +\sum_{k=0}^{n-2} \sum_{m=1}^{n-k-1}(-1)^{m} \frac{1}{m !}(\lambda \tau)^{m} e^{-(m+k) \lambda \tau}+\sum_{k=1}^{n-1} e^{-k \lambda \tau} .
\end{aligned}
$$

Using the distributive law, the right side of (4.1) equals

$$
\sum_{k=0}^{n-1} \sum_{m=0}^{n-1-k}(-1)^{k+1} \frac{1}{\lambda^{k}} \frac{(m+k) !}{m !} a_{n-1, m+k} \tau^{m} .
$$

For $k=m=0$ the summand is $-a_{n-1,0}$. For all $k, m$ with $m=n-1-k$ the summand is (using (2.6)):

$$
(-1)^{n-k-1}(\lambda \tau)^{n-1-k} \frac{1}{(n-1-k) !} e^{-(n-1) \lambda \tau} .
$$


Finally, for all $k, m$ with $1 \leq k+m \leq n-2$ the summand is (using (2.5)):

$$
(-1)^{m}(\lambda \tau)^{m} \frac{1}{m !} e^{-(m+k) \lambda \tau}\left(1-a_{n-m-k-1,0}\right) .
$$

Summing up (and using $(-1)^{n+k+1}=(-1)^{n-k-1}$ ), we get that the right side of (4.1) equals

$$
\begin{aligned}
& -a_{n-1,0} \\
& +\sum_{k=0}^{n-1}(-1)^{n-k-1} \frac{1}{(n-1-k) !}(\lambda \tau)^{n-1-k} e^{-(n-1) \lambda \tau} \\
& +\sum_{k=0}^{n-2} \sum_{m=1}^{n-k-2}(-1)^{m} \frac{1}{m !}(\lambda \tau)^{m} e^{-(m+k) \lambda \tau}\left(1-a_{n-m-k-1,0}\right) \\
& +\sum_{k=1}^{n-2} e^{-k \lambda \tau}\left(1-a_{n-k-1,0}\right) .
\end{aligned}
$$

The third term contains all $m, k$ with $1 \leq m+k \leq n-2$ and $m \geq 1$. The fourth term contains the remaining terms.

Taking the difference between (4.2) and (4.3) we get 0 .

Lemma 4.3. Let $n \in \mathbb{N}$ with $n \geq 2$. Then

$$
\sum_{k=0}^{n}(-1)^{k+1} \frac{1}{\lambda^{k}} p_{n}^{(k)}(0)=\sum_{k=0}^{n-1}(-1)^{k+1} \frac{1}{\lambda^{k}} p_{n-1}^{(k)}(\tau) .
$$

Proof. Combine Lemma 4.1 and Lemma 4.2 ,

Lemma 4.4. For all $n \in \mathbb{N}, n \geq 2$,

$$
a_{n, 0}=e^{-\lambda \tau}-\sum_{k=0}^{n}(-1)^{k+1} \frac{1}{\lambda^{k}} k ! a_{n, k}+\sum_{k=0}^{n-1}(-1)^{k+1} e^{-\lambda \tau} \frac{1}{\lambda^{k}} k ! a_{n-1, k} .
$$

Further, for all $m=1, \ldots, n$ we have

$$
a_{n, m}=-\sum_{k=0}^{n-m}(-1)^{k+1} \frac{1}{\lambda^{k}} \frac{(m+k) !}{m !} a_{n, m+k}+\sum_{k=0}^{n-1-m}(-1)^{k+1} e^{-\lambda \tau} \frac{1}{\lambda^{k}} \frac{(m+k) !}{m !} a_{n-1, m+k} .
$$

Proof. From (2.5) and (2.6) it follows easily that for each $n \geq 2$ and each $k=2, \ldots, n$ :

$$
a_{n-1, k-1}=-\frac{k}{\lambda} e^{\lambda \tau} a_{n, k} .
$$

Proof for $a_{n, 0}$ : The right side of the equation equals

$$
e^{-\lambda \tau}-\sum_{k=0}^{n}(-1)^{k+1} \frac{1}{\lambda^{k}} k ! a_{n, k}+\sum_{k=1}^{n}(-1)^{k} e^{-\lambda \tau} \frac{1}{\lambda^{k-1}}(k-1) ! a_{n-1, k-1} .
$$

By (4.4) the terms for $k \geq 2$ cancel each other. The remaining terms are

$$
e^{-\lambda \tau}+a_{n, 0}-\frac{1}{\lambda} a_{n, 1}-e^{-\lambda \tau} a_{n-1,0} .
$$

This equals $a_{n, 0}$, since by (2.5): $a_{n, 1}=\lambda e^{-\lambda \tau}\left(1-a_{n-1,0}\right)$.

Proof for $a_{n, m}$ : The case $n=m$ is trivial. For $m=1, \ldots, n-1$ we rewrite the right side as

$$
-\sum_{k=0}^{n-m}(-1)^{k+1} \frac{1}{\lambda^{k}} \frac{(m+k) !}{m !} a_{n, m+k}+\sum_{k=1}^{n-m}(-1)^{k} e^{-\lambda \tau} \frac{1}{\lambda^{k-1}} \frac{(m+k-1) !}{m !} a_{n-1, m+k-1} .
$$


For $k \geq 1$ we have $m+k \geq 2$. As above the terms for $k \geq 1$ cancel each other by (4.4) and the remaining term is (for $k=0$ ): $a_{n, m}$.

Lemma 4.5. We have for all $n \in \mathbb{N}, n \geq 2$ and $x \in \mathbb{R}$,

$$
\begin{aligned}
\sum_{k=0}^{n} a_{n, k} x^{k}= & e^{-\lambda \tau}-\sum_{m=0}^{n}\left(\sum_{k=0}^{n-m}(-1)^{k+1} \frac{1}{\lambda^{k}} \frac{(m+k) !}{m !} a_{n, m+k}\right) x^{m} \\
& +\sum_{m=0}^{n-1}\left(\sum_{k=0}^{n-1-m}(-1)^{k+1} e^{-\lambda \tau} \frac{1}{\lambda^{k}} \frac{(m+k) !}{m !} a_{n-1, m+k}\right) x^{m} .
\end{aligned}
$$

Proof. The coefficients of the polynomials on both sides of the equation coincide, as follows from Lemma 4.4 .

Lemma 4.6. We have for all $n \in \mathbb{N}, n \geq 2$ and $x \in \mathbb{R}$,

$$
p_{n}(x)=e^{-\lambda \tau}-\sum_{k=0}^{n}(-1)^{k+1} \frac{1}{\lambda^{k}} p_{n}^{(k)}(x)+e^{-\lambda \tau}\left(\sum_{k=0}^{n-1}(-1)^{k+1} \frac{1}{\lambda^{k}} p_{n-1}^{(k)}(x)\right) .
$$

Proof. Rearranging the terms in Lemma 4.5, we get

$$
\begin{aligned}
\sum_{k=0}^{n} a_{n, k} x^{k}= & e^{-\lambda \tau}-\sum_{k=0}^{n}(-1)^{k+1} \frac{1}{\lambda^{k}} \sum_{m=0}^{n-k}\left(\frac{(m+k) !}{m !} a_{n, m+k} x^{m}\right) \\
& +e^{-\lambda \tau}\left(\sum_{k=0}^{n-1}(-1)^{k+1} \frac{1}{\lambda^{k}} \sum_{m=0}^{n-1-k}\left(\frac{(m+k) !}{m !} a_{n-1, m+k} x^{m}\right)\right) .
\end{aligned}
$$

The claim now follows from Lemma 4.1

Next we derive a formula for solving the integral, which occurs in the Recursion Lemma.

Lemma 4.7. Let $\lambda, c \in \mathbb{R}, \lambda>0, n \in \mathbb{N}$, and let $p$ be a polynomial of degree $n$. Then

$$
\int p(x-y+c) \lambda e^{-\lambda y} d y=e^{-\lambda y}\left(\sum_{k=0}^{n}(-1)^{k+1} \frac{1}{\lambda^{k}} p^{(k)}(x-y+c)\right) .
$$

Proof. The formula can be proved by deriving the right side of the equation and the fact that $p^{(n+1)}=0(p$ is a polynomial of degree $n)$.

Proof of Lemma 3.4. Equation (3.4) follows from (2.2) and (2.3).

Now for (3.5): Using Lemma 4.7 we get

$$
\int_{0}^{x} p_{n}(x-y) \lambda e^{-\lambda y} d y=e^{-\lambda x}\left(\sum_{k=0}^{n}(-1)^{k+1} \frac{1}{\lambda^{k}} p_{n}^{(k)}(0)\right)-\left(\sum_{k=0}^{n}(-1)^{k+1} \frac{1}{\lambda^{k}} p_{n}^{(k)}(x)\right)
$$

and

$$
\begin{aligned}
& \int_{x}^{\tau} p_{n-1}(x-y+\tau) \lambda e^{-\lambda y} d y \\
& \quad=e^{-\lambda \tau}\left(\sum_{k=0}^{n-1}(-1)^{k+1} \frac{1}{\lambda^{k}} p_{n-1}^{(k)}(x)\right)-e^{-\lambda x}\left(\sum_{k=0}^{n-1}(-1)^{k+1} \frac{1}{\lambda^{k}} p_{n-1}^{(k)}(\tau)\right) .
\end{aligned}
$$

Now, Lemma 4.3 and Lemma 4.6 show (3.5).

We have

$$
p_{n}(0)=a_{n, 0}
$$


and

$$
p_{n-1}(\tau)=\sum_{k=0}^{n-1} a_{n-1, k} \tau^{k}
$$

We apply (2.5) and (2.6) on every $a_{n-1, k}$ with $k=1, \ldots, n-1$ in (4.7) and get the right side of (2.4). This shows $p_{n}(0)=p_{n-1}(\tau)$.

\section{BIBLIOGRAPHY}

1. S. Asmussen, Applied Probability and Queues, Wiley, New York, 1987. MR889893 (89a:60208)

2. E. Grycko and O. Moeschlin, A criterion for the occurrence or non-occurrence of a traffic collapse at a bottleneck, Commun. Statist. Stochastic Models 14 (1998), no. 3, 571-584. MR 1621330 (99g:90042)

3. E. Grycko and O. Moeschlin, A concept of optimal control at a bottleneck with symmetric volume of traffic, Commun. Stat. Stochastic Models 14 (1998), no. 3, 585-600. MR1621334 (99g:90043)

4. S. P. Meyn and R. L. Tweedie, Markov Chains and Stochastic Stability, 2nd. Ed., Springer, London, 1993. MR.1287609 (95j:60103)

5. F. Recker, On the asymptotical queue length in vehicular traffic confluence (2005). (to appear)

Department of Mathematics, University of Hagen, D-58084 Hagen, Germany

E-mail address: frank.recker@fernuni-hagen.de

Received 3/OCT/2005

Originally published in English 